\title{
Synthetic MRI in the Detection of Multiple Sclerosis Plaques
}

\author{
(D) A. Hagiwara, (D) M. Hori, DK. Yokoyama, (D).Y. Takemura, (DC. Andica, (D). Tabata, DK. Kamagata, (D). Suzuki, \\ (D).K. Kumamaru, DM. Nakazawa, (D) N. Takano, DH. Kawasaki, DN. Hamasaki, (D) A. Kunimatsu, and (D). Aoki
}

\begin{abstract}
BACKGROUND AND PURPOSE: Synthetic MR imaging enables the creation of various contrast-weighted images including double inversion recovery and phase-sensitive inversion recovery from a single MR imaging quantification scan. Here, we assessed whether synthetic MR imaging is suitable for detecting MS plaques.
\end{abstract}

MATERIALS AND METHODS: Quantitative and conventional MR imaging data on 12 patients with MS were retrospectively analyzed Synthetic T2-weighted, FLAIR, double inversion recovery, and phase-sensitive inversion recovery images were produced after quantification of $\mathrm{T} 1$ and $\mathrm{T} 2$ values and proton density. Double inversion recovery images were optimized for each patient by adjusting the TI. The number of visible plaques was determined by a radiologist for a set of these 4 types of synthetic MR images and a set of conventional T1-weighted inversion recovery, T2-weighted, and FLAIR images. Conventional 3D double inversion recovery and other available images were used as the criterion standard. The total acquisition time of synthetic MR imaging was 7 minutes 12 seconds and that of conventional MR imaging was 6 minutes 29 seconds The lesion-to-WM contrast and lesion-to-WM contrast-to-noise ratio were calculated and compared between synthetic and conventional double inversion recovery images.

RESULTS: The total plaques detected by synthetic and conventional MR images were 157 and 139, respectively $(P=.014)$. The lesionto-WM contrast and contrast-to-noise ratio on synthetic double inversion recovery images were superior to those on conventional double inversion recovery images $(P=.001$ and $<0.001$, respectively)

CONCLUSIONS: Synthetic MR imaging enabled detection of more MS plaques than conventional MR imaging in a comparable acquisition time. The contrast for MS plaques on synthetic double inversion recovery images was better than on conventional double inversion recovery images.

ABBREVIATIONS: CNR = contrast-to-noise ratio; $\mathrm{DIR}=$ double inversion recovery; $\mathrm{PD}=$ proton density; $\mathrm{PSIR}=$ phase-sensitive inversion recovery; $\mathrm{QRAPMASTER}=$ quantification of relaxation times and proton density by multiecho acquisition of saturation recovery with TSE readout; $\mathrm{SI}=$ signal intensity; TIIR = TI-weighted inversion recovery

$\mathbf{M}^{\mathrm{s}}$ is a CNS demyelination disorder that usually strikes young adults. MR imaging serves an important role in MS diagnosis and surveillance via the detection and follow-up of focal and diffuse CNS lesions. Monitoring new or enlarging MS plaques is suitable for following disease activity when evaluating treat-

Received June 27, 2016; accepted after revision September 20

From the Departments of Radiology (A.H., M.H., M.Y.T., C.A., T.T., K.K., M.S., K.K.K., M.N., N.T., H.K., N.H., S.A.) and Neurology (K.Y.), Juntendo University School of Medicine, Tokyo, Japan; Department of Radiology (A.H., A.K.), Graduate School of Medicine, The University of Tokyo, Tokyo, Japan; and Department of Radiological Sciences (M.N.), Graduate School of Human Health Sciences, Tokyo Metropolitan University, Tokyo, Japan.

This work was supported by the Japan Society for the Promotion of Science KAKENHI, grant number 16K19852; and grants from the Ministry of Education, Culture, Sports, Science and Technology-Supported Program for the Strategic Research Foundation at Private Universities (2011-2015); and it was funded by the Impulsing Paradigm Change through disruptive Technologies (ImPACT) Program of the Council for Science, Technology and Innovation (Cabinet Office, Government of Japan). ment effects. ${ }^{1}$ The number of lesions detected early in the disease process is associated with future relapse, disability accumulation, or cognitive deficits. ${ }^{2}$ Several reports have shown the utility of double inversion recovery (DIR) and phase-sensitive inversion recovery (PSIR) images for detecting MS plaques, especially in intracortical or mixed WM-GM areas. ${ }^{3-6}$ Relative to FLAIR and T2-weighted images, DIR suppresses WM and CSF signals, thereby increasing the conspicuity of lesions in both GM and WM. ${ }^{7}$ PSIR is a T1-weighted inversion recovery (T1IR) sequence with phase-sensitive reconstruction that provides a greater dynamic range of signal intensity (SI) and higher tissue contrast than

Please address correspondence to Akifumi Hagiwara, MD, Department of Radiology, The University of Tokyo, 7-3-1, Hongo, Bunkyo-ku, Tokyo, Japan, 113-8655; e-mail: ahagiwara-tky@umin.ac.jp

- Indicates open access to non-subscribers at www.ajnr.org

http://dx.doi.org/10.3174/ajnr.A5012 
conventional T1IR sequences by additively combining positive and negative longitudinal magnetization. ${ }^{8}$ However, the additional acquisition time required has hindered the clinical use of DIR and PSIR images.

Synthetic MR imaging enables the production of images with almost any contrast-weighting, including DIR and PSIR, by virtually adjusting the TR, TE, and TI after quantifying the longitudinal T1 and transverse T2 relaxation times and the proton density (PD). ${ }^{9,10}$ Quantification of relaxation times and proton density by multiecho acquisition of saturation recovery with TSE readout (QRAPMASTER), an MR imaging quantification pulse sequence, was recently introduced into the clinical setting and has greatly shortened the time required for quantifying these parameters. ${ }^{11}$ A recent phantom study revealed that T1, T2, and PD measurements made with the QRAPMASTER pulse sequence were sufficiently accurate and reproducible. ${ }^{12}$ Synthetic MR imaging of the brain generates images inferior in quality but comparable in diagnostic power with those acquired by conventional MR imaging. ${ }^{13}$ Synthetic MR imaging is reported to be useful in evaluating brain metastases ${ }^{14}$ and Sturge-Weber syndrome. ${ }^{15,16}$ Moreover, some reports have demonstrated the utility of synthetic MR imaging in evaluating patients with MS. ${ }^{17-19}$ However, the added value of synthetic PSIR and DIR to detect MS plaques remains to be examined. The research described above suggests that generating PSIR and DIR images in addition to T2-weighted and FLAIR images may boost the detectability of MS plaques by MR imaging.

Here, we assessed the usefulness of synthetic MR imaging, which enables creation of any contrast-weighted images including PSIR and DIR, for detecting focal MS plaques by comparing images obtained from synthetic and conventional MR imaging.

\section{MATERIALS AND METHODS Study Participants}

The study was approved by the institutional review board of Juntendo University Hospital, Tokyo, Japan. Given its retrospective nature, written informed consent was not required. Data from 22 consecutive patients with MS who had undergone quantitative and conventional MR imaging from August 2015 through November 2015 were retrospectively reviewed. These patients were diagnosed according to standard criteria. ${ }^{20-22}$ Of the 22 patients, 10 had diffusely abnormal white matter ${ }^{23}$ and were excluded from the study because the number of plaques was difficult to count accurately. Therefore, data from 12 patients ( 1 male and $11 \mathrm{fe}-$ males; mean age, 35.5 years; age range, $16-50$ years) were evaluated. Of the 12 patients, 10 had relapsing-remitting MS and 2 had clinically isolated syndrome. The median score on the Expanded Disability Status Scale ${ }^{24}$ at imaging was zero (range, 0-3.0), and the mean disease duration was $6.2 \pm 5.1$ years.

\section{MR Imaging}

For all patients, MR imaging was performed on a 3T scanner (Discovery MR750w; GE Healthcare, Milwaukee, Wisconsin) with a 12-channel head coil. All patients underwent quantitative 2D axial imaging and conventional 2D axial T1IR, T2-weighted, FLAIR, and 3D sagittal DIR imaging.
Quantitative MR imaging was performed by using the 2D axial QRAPMASTER pulse sequence. ${ }^{11}$ This is a multisection, multiecho, multisaturation delay saturation-recovery turbo spin-echo acquisition method in which images are collected for different combinations of TEs and saturation delay times. At our institution, 2 TEs and 4 delay times were used to generate 8 real images and 8 imaginary ones, which were then used to quantify longitudinal $\mathrm{T} 1$ and transverse $\mathrm{T} 2$ relaxation times and the PD. The TEs used were 16.9 and $84.5 \mathrm{~ms}$, and the delay time was set as defined by the manufacturer of SyMRI software (SyntheticMR, Linköping, Sweden). The parameters used for quantitative MR imaging were as follows: FOV, $240 \times 240 \mathrm{~mm}$; matrix, $320 \times 320$; echo-train length, 10; bandwidth, $31.25 \mathrm{kHz}$; section thickness/gap, $4.0 \mathrm{~mm} /$ $1.0 \mathrm{~mm}$; and sections, 30 . The data acquired at each section were used to produce T1, T2, and PD maps, which were then used to calculate the synthetic MR images. Quantification map acquisition and raw data processing were performed with SyMRI software (Version 8.0; SyntheticMR).

The parameters used to produce synthetic T2-weighted images were TR, $4500 \mathrm{~ms}$; and TE, $100 \mathrm{~ms}$. Those used to generate FLAIR images were TR, 15,000 ms; TE, $100 \mathrm{~ms}$; and TI, $2900 \mathrm{~ms}$. Finally, those used to obtain PSIR images were TR, $6000 \mathrm{~ms}$; TE, $15 \mathrm{~ms}$; and TI, $500 \mathrm{~ms}$. The parameters for DIR image synthesis, which were optimized for each patient, are shown in the following section.

The parameters used to obtain conventional T1IR images were the following: TR, $3294 \mathrm{~ms}$; TE, $18 \mathrm{~ms}$; TI, $908 \mathrm{~ms}$; FOV, $240 \times$ $216 \mathrm{~mm}$; matrix, $352 \times 256$; echo-train length, 8 ; section thickness/gap, $4 \mathrm{~mm} / 1 \mathrm{~mm}$; and sections, 30 . Those used to obtain T2-weighted images were the following: TR, $4500 \mathrm{~ms}$; TE, $111 \mathrm{~ms}$; FOV, $240 \times 240 \mathrm{~mm}$; matrix, $512 \times 512$; echo-train length, 24 ; section thickness/gap, $4 \mathrm{~mm} / 1 \mathrm{~mm}$; and sections, 30 . Those used to obtain FLAIR images were the following: TR, $9000 \mathrm{~ms}$; TE, 124 ms; FOV, $240 \times 240 \mathrm{~mm}$; matrix, $320 \times 224$; echo-train length, 16; section thickness/gap, $4 \mathrm{~mm} / 1 \mathrm{~mm}$; and sections, 30. Finally, those used to produce 3D sagittal DIR images were the following: TR, $7000 \mathrm{~ms}$; TE, $90 \mathrm{~ms}$; first TI, $2892 \mathrm{~ms}$; second TI, $546 \mathrm{~ms}$; FOV, $256 \times 256$ mm; matrix, $192 \times 192$; echo-train length, 160; section thickness, $1.0 \mathrm{~mm}$; and sections, 160 . The first TI was defined as the interval between the first $180^{\circ}$ inversion pulse and the $90^{\circ}$ excitation pulse. The second TI was defined as the interval between the second $180^{\circ}$ inversion pulse and the $90^{\circ}$ excitation pulse.

The acquisition time was 7 minutes 12 seconds for quantitative MR imaging, 1 minute 50 seconds for conventional T1IR, 2 minutes 6 seconds for conventional T2-weighted, 2 minutes 33 seconds for conventional FLAIR, and 6 minutes 15 seconds for conventional 3D DIR images. These images were saved as DICOM files and analyzed with OsiriX Imaging Software, Version 7.0 (http:// www.osirix-viewer.com) on a personal computer.

\section{Synthetic DIR Optimization}

The parameters for creating synthetic DIR images were optimized for each patient by adjusting the second TI to intensify the contrast between MS plaques and WM (Fig 1). All other parameters were the same for each patient, as follows: TR, 15,000 ms; TE, 100 ms; and first TI (TI1), $3750 \mathrm{~ms}$. The second TI (TI2) was selected to suppress the WM and CSF signals while maximizing the GM 


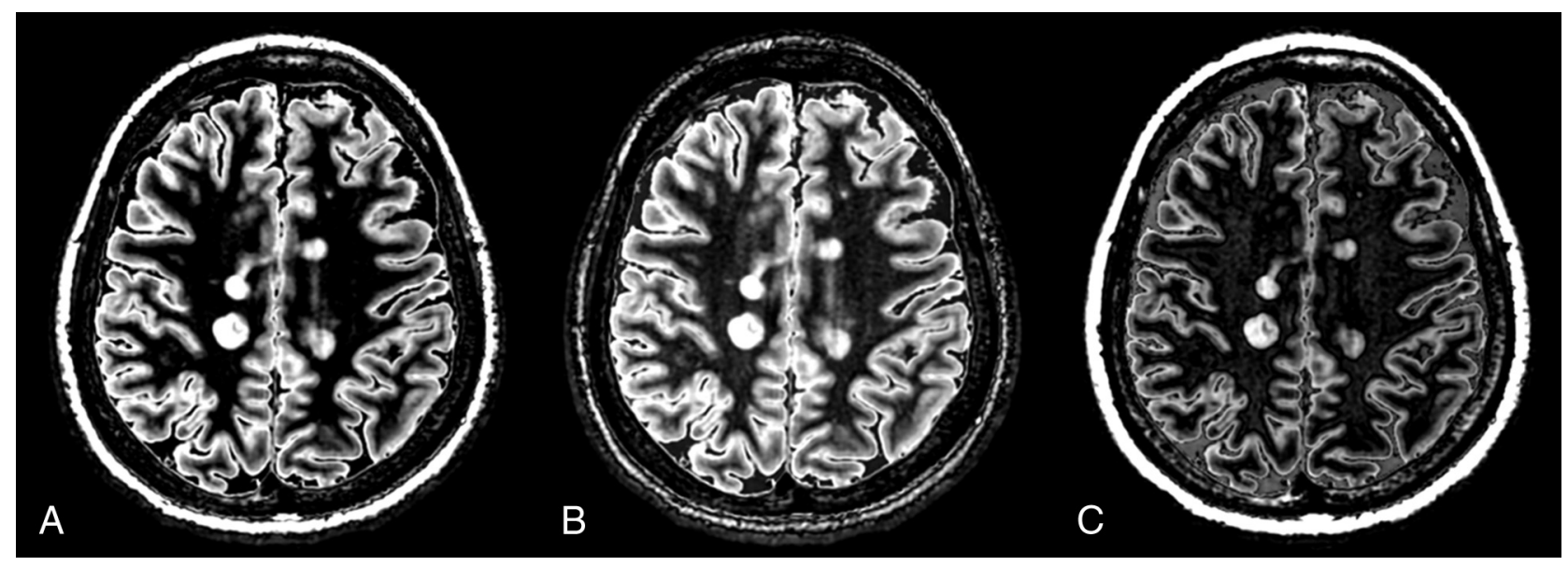

FIG 1. An example of DIR optimization. A DIR image with a second TI of $460 \mathrm{~ms}(A)$ (as determined according to the equations in the main text) shows better delineation of MS plaques than a DIR image with a second TI of $360 \mathrm{~ms}(B)$ or $560 \mathrm{~ms}$ (C).

signal, in accordance with the method of Gabr et al. ${ }^{25}$ This procedure was accomplished by minimizing the following expression:

$$
\frac{S I_{\mathrm{WM}}+S I_{\mathrm{CSF}}}{S I_{\mathrm{GM}}}
$$

where $S I_{\mathrm{WM}}, S I_{\mathrm{CSF}}$, and $S I_{\mathrm{GM}}$ denote the signal intensities (SIs) of WM, CSF, and GM, respectively. For each tissue type, we measured T1, T2, and PD on SyMRI software to estimate the SI by using the following expression, modified from the work of Redpath and Smith ${ }^{26}$ :

$$
\begin{aligned}
S I & =P D\{1-2 \exp (-T I 2 / T 1)+2 \exp [-(T I 1+T I 2) / T 1] \\
& -\exp (-T R / T 1)[2 \exp (T E / 2 T 1)-1]\}(\exp (-T E / T 2)) .
\end{aligned}
$$

Here, T1, T2, and PD of each tissue type were averaged from the following ROIs $(3 \times 3$ voxels, corresponding to $2.25 \times 2.25$ $\mathrm{mm}^{2}$ ): CSF, bilaterally in the anterior horns of the lateral ventricles; GM, bilaterally in the thalamus, occipital cortex, and frontal cortex; and WM, in the corpus callosum-one in the genu and another in the splenium-and bilaterally in the centrum semiovale. The second TI of each image fell in the range of $460-480 \mathrm{~ms}$ after optimization of the synthetic DIR images for each patient.

\section{Image Analysis}

Qualitative Analysis of Conventional and Synthetic Images. An experienced neuroradiologist (K.K.), blinded to the clinical information, individually counted the number of lesions in the synthetic and conventional image sets, which were shown in random order in 1 session. One synthetic image set included T2-weighted, FLAIR, DIR, and PSIR images for each patient (Fig 2). One conventional image set included T2-weighted, FLAIR, and T1IR images. Lesions at least $3 \mathrm{~mm}$ in diameter were counted. To confirm the accuracy of lesion counts, an experienced neuroradiologist (M.S.) also independently conducted MR imaging evaluations of the same imaging datasets. Conventional 3D DIR images and all other images were used as criterion standards by an experienced neuroradiologist (A.H.) to determine whether a counted lesion was a true- or false-positive and to classify the lesion according to its location as an infratentorial, deep GM, periventricular WM, deep WM, juxtacortical WM, or mixed WM-GM lesion. If a lesion was detected only on the conventional image set or the synthetic image set but not on conventional 3D DIR images, we defined it as a false-positive.

Quantitative Analysis of Conventional and Synthetic DIR Images.

The lesion-to-WM contrast and the contrast-to-noise ratio (CNR) were among the indices used to quantify synthetic and conventional DIR images. To assess image quality, we also analyzed the GM-to-WM contrast, GM-to-CSF contrast, and CNR. To match the section thickness between synthetic and conventional DIR images, we reconstructed conventional 2D axial DIR images (thickness, $4 \mathrm{~mm}$; gap, $1 \mathrm{~mm}$ ) from conventional 3D DIR sagittal images.

ROI analyses were performed on synthetic and conventional DIR images by a single investigator (M.N.) blinded to the clinical information. For the quantitative analysis, the signal intensities of the MS lesions and corresponding WM were measured by ROI analyses and their mean values were recorded. A circular ROI that covered almost the entire lesion was placed on each MS plaque that measured $\geq 5 \mathrm{~mm}$ in diameter. The ROI was then copied and pasted on the corresponding WM (Fig 3). For a supratentorial lesion, the corresponding WM was defined as the normal-appearing WM contralateral to that lesion. For an infratentorial lesion, it was defined as the normal-appearing WM in the brain stem in the same section.

In synthetic MR imaging, the SI of the surrounding air is set to zero. Hence, the median SD of the SIs of the following 12 ROIs, all of which were $2.4 \times 2.4 \mathrm{~mm}^{2}$, was defined as the noise for each patient: ROIs in the CSF (bilaterally in the anterior horns of the lateral ventricles), in the GM (bilaterally in the thalamus, occipital cortex, and frontal cortex), and in the WM (in the corpus callosum-one in the genu and another in the splenium —and bilaterally in the centrum semiovale). This approach was a modified version of the method used by Blystad et al. ${ }^{13}$

To assess the effect of optimizing WM signal suppression on the image quality of DIR images, we compared the SI of GM $\left(\mathrm{SI}_{\mathrm{GM}}\right)$ with the SIs of WM $\left(\mathrm{SI}_{\mathrm{WM}}\right)$ and $\mathrm{CSF}\left(\mathrm{SI}_{\mathrm{CSF}}\right) \cdot \mathrm{SI}_{\mathrm{GM}}, \mathrm{SI}_{\mathrm{WM}}$, and $\mathrm{SI}_{\mathrm{CSF}}$ were obtained by averaging the values of the 6 , 4 , and 2 regions above, respectively, for each patient.

AJNR Am J Neuroradiol 38:257-63 Feb 2017 www.ajnr.org 


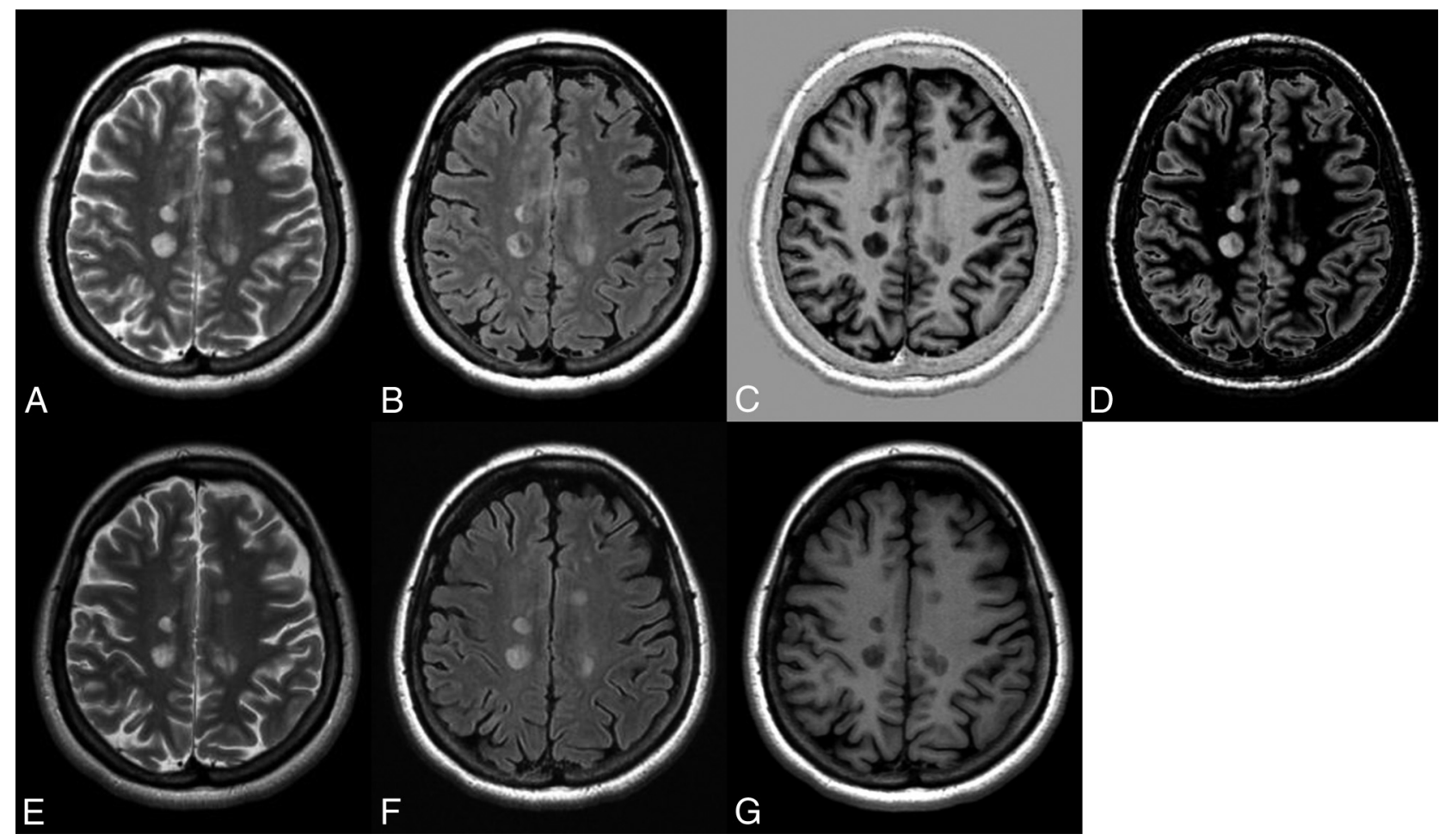

FIG 2. Representative sections of synthetic T2-weighted (A), FLAIR (B), PSIR (C), and DIR $(D)$ images, along with conventional T2-weighted $(E)$, FLAIR $(F)$, and TIIR $(G)$ images.

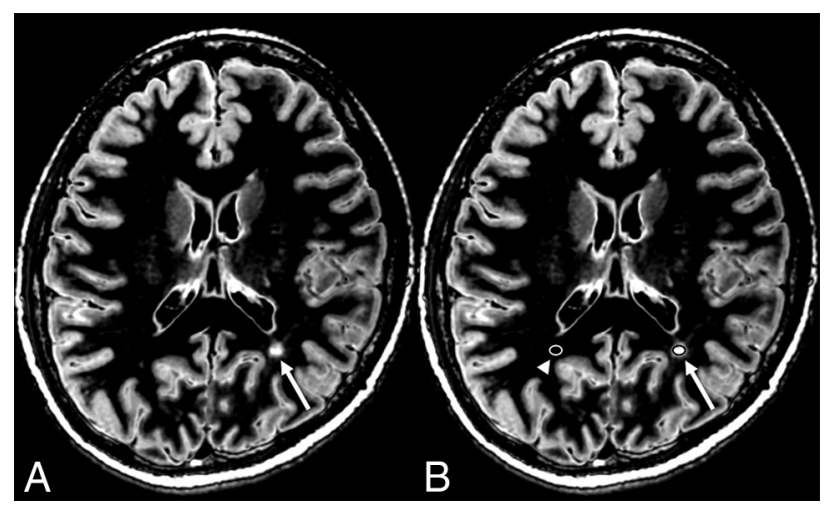

FIG 3. An example of ROI placement. A plaque (arrow) is shown on the synthetic DIR image (A). A circular ROI (arrow) that covers almost the entire lesion is placed in $B$. The ROI was copied and pasted on the contralateral normal-appearing WM (arrowhead).

The lesion-to-WM contrast was defined as the difference between the mean SI of a lesion and that of the corresponding WM divided by the SI of the corresponding WM. The lesion-to-WM CNR was defined as the difference between the SI of a lesion and that of the corresponding WM divided by the noise of each sequence. The GM-to-WM and GM-to-CSF contrasts and CNRs were calculated likewise.

\section{Statistical Analysis}

Statistics were computed by using the free software R, Version 3.2.1 (R statistical and computing software; http://www.r-project. org/). Because none of the datasets were normally distributed, we used the nonparametric Wilcoxon signed rank test to compare the number of lesions detected in the synthetic and conventional im-
Table 1: Multiple sclerosis plaques detected by a neuroradiologist on synthetic and conventional MR images

\begin{tabular}{lccc}
\hline \multicolumn{1}{c}{ Region } & $\begin{array}{c}\text { Synthetic } \\
\text { MRI (No.) }\end{array}$ & $\begin{array}{c}\text { Conventional } \\
\text { MRI (No.) }\end{array}$ & $\begin{array}{c}\boldsymbol{P} \\
\text { Value }\end{array}$ \\
\hline Infratentorial & 2 & 2 & 1 \\
Periventricular WM & 31 & 28 & .374 \\
Deep WM & 87 & 82 & .198 \\
Juxtacortical WM & 25 & 20 & .547 \\
Mixed WM-GM & 6 & 5 & .773 \\
Deep GM & 6 & 2 & .203 \\
Total & 157 & 139 & .014 \\
False-positives & 3 & 1 & .586 \\
\hline
\end{tabular}

ages as well as the contrasts and CNRs of synthetic and conventional DIR images. A 2 -sided $P$ value $<.05$ was considered significant.

\section{RESULTS}

The total number of lesions detected by K.K. on synthetic images was significantly larger than that on conventional images (Table 1). However, no significant difference was detected in any individual region. Several lesions were easier to find on synthetic DIR or PSIR images than on conventional images and were detected only on the synthetic image set but not on the conventional image set by K.K. (Fig 4). All false-positives on synthetic images were located next to the CSF (Fig 5), because the surface of the brain tended to become hyperintense on synthetic FLAIR and DIR images.

The interobserver reproducibility between the 2 observers (K.K. and M.S.) for the total number of detected lesions was then measured. The interclass correlation coefficient of synthetic MR imaging was 0.858 (95\% CI, 0.496-0.959). That of conventional MR imaging was 0.950 (95\% CI, 0.824-0.986). 


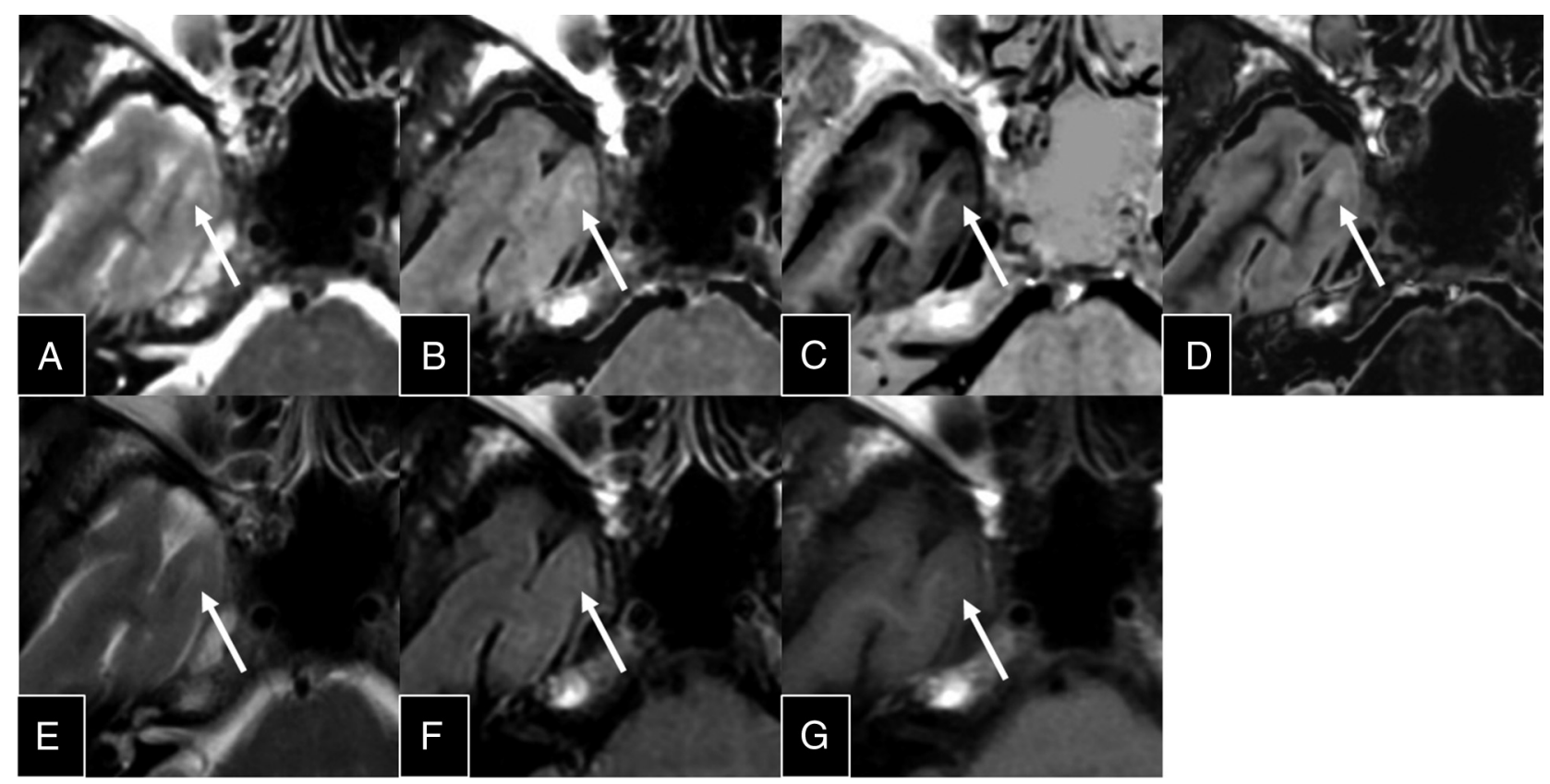

FIG 4. An MS plaque readily detected on PSIR and DIR images. This plaque (arrows) is difficult to identify on synthetic T2-weighted ( $A$ ) and FLAIR $(B)$ images and on conventional T2-weighted $(E)$, FLAIR $(F)$, and TIIR $(G)$ images, but it is clearly delineated on synthetic PSIR $(C)$ and DIR $(D)$ images. The plaque was detected by a neuroradiologist (K.K.) on synthetic but not conventional MR images.

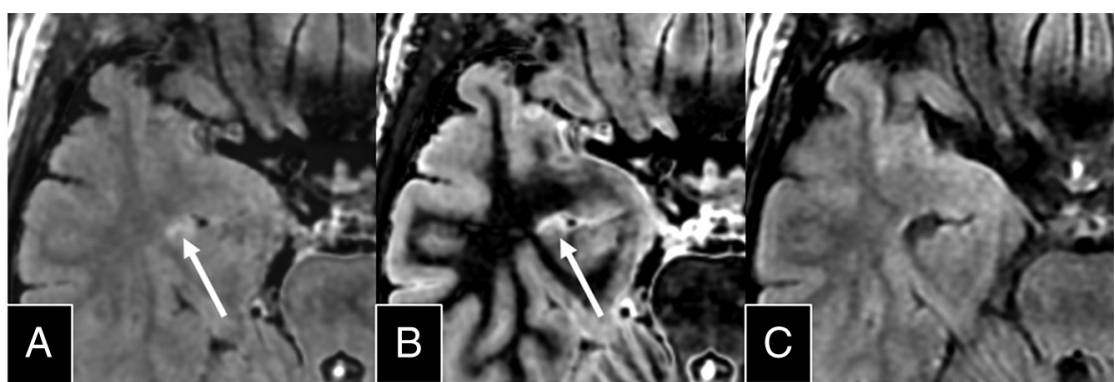

FIG 5. An example of a false-positive lesion. The surface of the brain tends to become hyperintense on synthetic FLAIR and DIR images. Note the hyperintense focus (arrow) in the inferior horn of the right lateral ventricle on synthetic FLAIR $(A)$ and DIR (B) images; this focus was identified as an MS plaque by a neuroradiologist (K.K.). No hyperintense focus is seen in the same place on a conventional FLAIR image $(C)$.

Table 2: Contrast and CNR among lesions, WM, GM, and CSF

\begin{tabular}{lccr}
\hline & $\begin{array}{c}\text { Synthetic } \\
\text { DIR }^{\mathbf{a}}\end{array}$ & $\begin{array}{c}\text { Conventional } \\
\text { DIR }^{\mathbf{a}}\end{array}$ & $\begin{array}{c}\boldsymbol{P} \\
\text { Value }\end{array}$ \\
\hline Lesion-to-WM contrast & $9.33 \pm 5.92$ & $6.74 \pm 3.58$ & .001 \\
Lesion-to-WM CNR & $23.50 \pm 7.90$ & $20.20 \pm 8.30$ & $<.001$ \\
GM-to-WM contrast & $5.21 \pm 2.26$ & $3.73 \pm 1.18$ & .027 \\
GM-to-WM CNR & $13.07 \pm 2.77$ & $11.10 \pm 3.89$ & .012 \\
GM-to-CSF contrast & $17.05 \pm 20.30$ & $3.98 \pm 1.18$ & $<.001$ \\
GM-to-CSF CNR & $14.33 \pm 2.68$ & $11.17 \pm 3.61$ & .009 \\
\hline
\end{tabular}

${ }^{a}$ Values are means $\pm S D$.

The lesion-to-WM, GM-to-WM, and GM-to-CSF contrasts and their corresponding CNRs of synthetic DIR images were significantly higher than those of conventional DIR images (Table 2 and Fig 6).

\section{DISCUSSION}

Synthetic MR imaging enabled us to detect more MS plaques than conventional MR imaging in a comparable acquisition time (7 minutes 12 seconds versus 6 minutes 29 seconds). Previous re- ports have shown that DIR and PSIR images are superior to T2-weighted and FLAIR images for detecting intracortical or mixed WM-GM lesions ${ }^{3,4,6}$; these reports were of MR images of $\leq 3-\mathrm{mm} \mathrm{sec}$ tion thickness. However, the ability to detect mixed WM-GM plaques did not differ significantly between synthetic and conventional MR images in our study, which were obtained with a section thickness of $4 \mathrm{~mm}$. In a study of DIR images with a section thickness of $5 \mathrm{~mm}$, significantly more MS plaques were detected on DIR images than on FLAIR and T2-weighted images, but not in the case of mixed WM-GM lesions. ${ }^{5}$ Likewise, our use of a section thickness of $4 \mathrm{~mm}$ and a small number of patients may have hindered the detection of more mixed WM-GM lesions on synthetic than on conventional MR imaging. Including more patients in our study may have enabled the detection of more intracortical or mixed WM-GM plaques on synthetic than on conventional MR imaging.

All the false-positives on synthetic images were located next to the CSF (Fig 5). In synthetic FLAIR and DIR images, the surface of the brain tends to become hyperintense, which is presumably caused by the partial volume effect. The surface of the brain is even brighter on synthetic DIR images than on synthetic FLAIR images (Fig 2). This feature may not be a problem if the existence of these artifacts is known in advance of reading synthetic MR images.

In this study, even though the interclass correlation coefficient of synthetic MR imaging between the 2 observers was excellent, it was lower and had a wider range than that of conventional MR imaging. One explanation is that these 2 readers had never been 


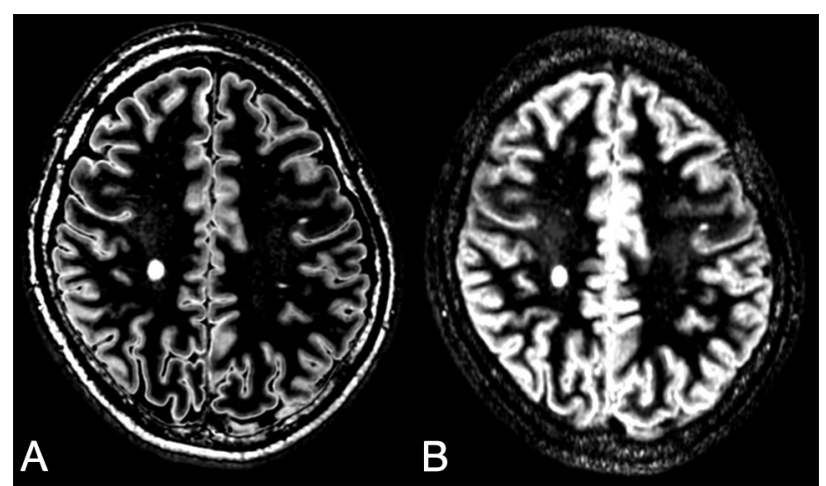

FIG 6. Sample sections of synthetic $(A)$ and conventional ( $B)$ DIR images. Note the better suppression of WM on the synthetic DIR image.

exposed to synthetic MR imaging prior to this study. After the readers become accustomed to synthetic MR imaging, the agreement rate may be higher.

The quality of DIR images was better in synthetic MR imaging (which was optimized for each patient) than in conventional MR imaging. No previous report has evaluated the image quality of synthetic DIR. Synthetic T1-weighted and T2-weighted images were reported to have higher contrast and comparable CNR compared with conventional images. ${ }^{13}$ Synthetic FLAIR images were reported to have lower contrast and lower CNR than conventional FLAIR images. In our study, we succeeded in creating synthetic DIR images with higher contrast and CNR than conventional DIR images by optimizing them for each patient. This result shows the potential advantage of synthetic MR imaging, which can be optimized for each patient after image acquisition. A recent report attempted to optimize the DIR image for each patient. ${ }^{25}$ In this report, DIR images were acquired after obtaining T1 and T2 maps separately and then optimizing the acquisition parameters according to these maps. Because quantification and image creation can be completed in a single acquisition and on the same software, the method used in our study has the potential to reduce the time needed for DIR optimization.

Our study had a number of limitations. First, the sample size was small. Second, the section thickness of the MR images acquired was $4 \mathrm{~mm}$ with a 1-mm gap. This thickness was due to the technical problem of not being able to obtain synthetic MR images without a gap of at least $1 \mathrm{~mm}$ to reduce cross-talk between sections, even though current imaging guidelines for MS recommend a section thickness of $\leq 3 \mathrm{~mm}$ without a gap for $2 \mathrm{D}$ acquisition. ${ }^{27,28}$ This problem should be addressed in future studies. Third, the resolution was not the same for all sequences. The retrospective nature of this study hindered the parameter adjustment.

\section{CONCLUSIONS}

Synthetic MR imaging enabled the detection of more MS plaques than conventional MR imaging in a comparable acquisition time, owing to the creation of useful contrast-weighted images (ie, DIR, PSIR) not acquired routinely on conventional MR imaging. The quality of DIR images in synthetic MR imaging optimized for each patient was superior to that in conventional MR imaging. Our results show that synthetic MR imaging has the potential to be useful for detecting MS plaques.
Disclosures: Akifumi Hagiwara—RELATED: Grant:Japan Society for the Promotion of Science; a Ministry of Education, Culture, Sports, Science and Technology-Supported Program; and the Impulsing Paradigm Change through disruptive Technologies (ImPACT) program, Comments: Japan Society for the Promotion of Science. KAKENHI, grant number 16K19852; a Ministry of Education, Culture, Sports, Science and Technology-Supported Program for the Strategic Research Foundation at Private Universities (2011-2015); ImPACT program of the Council for Science, Technology and Innovation. Nao TakanoUNRELATED: Payment for Lectures including Service on Speakers Bureaus: GE Healthcare Japan, Comments: The 42nd Japanese Society of Radiological Technology Autumn Scientific Congress, October 9-11, 2014, Sapporo, Hokkaido, Japan; luncheon seminar concerning experience in MR angiography (silent MRA) using the Silenz sequence. Akira Kunimatsu-UNRELATED: Payment for Lectures including Service on Speakers Bureaus: Siemens Healthcare K.K., Toshiba Medical Systems, Terumo, Philips Electronics Japan; Payment for Manuscript Preparation: Bayer Yakuhin, GE Healthcare Japan.

\section{REFERENCES}

1. Bonzano L, Roccatagliata L, Mancardi GL, et al. Gadolinium-enhancing or active T2 magnetic resonance imaging lesions in multiple sclerosis clinical trials? Mult Scler 2009;15:1043-47 CrossRef Medline

2. Lavery AM, Verhey LH, Waldman AT. Outcome measures in relapsing-remitting multiple sclerosis: capturing disability and disease progression in clinical trials. Mult Scler Int 2014;2014:262350 CrossRef Medline

3. Geurts JJ, Pouwels PJ, Uitdehaag BM, et al. Intracortical lesions in multiple sclerosis: improved detection with 3D double inversionrecovery MR imaging. Radiology 2005;236:254-60 Medline

4. Nelson F, Poonawalla AH, Hou P, et al. Improved identification of intracortical lesions in multiple sclerosis with phase-sensitive inversion recovery in combination with fast double inversion recovery MR imaging. AJNR Am J Neuroradiol 2007;28:1645-49 Medline

5. Wattjes MP, Lutterbey GG, Gieseke J, et al. Double inversion recovery brain imaging at 3T: diagnostic value in the detection of multiple sclerosis lesions. AJNR Am J Neuroradiol 2007;28:54-59 Medline

6. Sethi V, Yousry TA, Muhlert N, et al. Improved detection of cortical MS lesions with phase-sensitive inversion recovery MRI. J Neurol Neurosurg Psychiatry 2012;83:877-82 CrossRef Medline

7. Bedell BJ, Narayana PA. Implementation and evaluation of a new pulse sequence for rapid acquisition of double inversion recovery images for simultaneous suppression of white matter and CSF. $J$ Magn Reson Imaging 1998;8:544-47 Medline

8. Hou P, Hasan KM, Sitton CW, et al. Phase-sensitive T1 inversion recovery imaging: a time-efficient interleaved technique for improved tissue contrast in neuroimaging. AJNR Am J Neuroradiol 2005;26:1432-38 Medline

9. Bobman SA, Riederer SJ, Lee JN, et al. Cerebral magnetic resonance image synthesis. AJNR Am J Neuroradiol 1985;6:265-69 Medline

10. Riederer SJ, Suddarth SA, Bobman SA, et al. Automated MR image synthesis: feasibility studies. Radiology 1984;153:203-06 Medline

11. Warntjes JB, Leinhard OD, West J, et al. Rapid magnetic resonance quantification on the brain: optimization for clinical usage. Magn Reson Med 2008;60:320-29 Medline

12. Krauss W, Gunnarsson M, Andersson T, et al. Accuracy and reproducibility of a quantitative magnetic resonance imaging method for concurrent measurements of tissue relaxation times and proton density. Magn Reson Imaging 2015;33:584-91 CrossRef Medline

13. Blystad I, Warntjes JB, Smedby O, et al. Synthetic MRI of the brain in a clinical setting. Acta Radiol 2012;53:1158-63 CrossRef Medline

14. Hagiwara A, Hori M, Suzuki M, et al. Contrast-enhanced synthetic MRI for the detection of brain metastases. Acta Radiol Open 2016;5: 2058460115626757 CrossRef Medline

15. Hagiwara A, Nakazawa M, Andica C, et al. Dural enhancement in a patient with Sturge-Weber syndrome revealed by double inversion recovery contrast using synthetic MRI. Magn Reson Med Sci 2016; 15:151-52 CrossRef Medline

16. Andica C, Hagiwara A, Nakazawa M, et al. The advantage of synthetic MRI for the visualization of early white matter change in an infant with Sturge-Weber syndrome. Magn Reson Med Sci 2016;15: 347-48 CrossRef Medline 
17. West J, Aalto A, Tisell A, et al. Normal appearing and diffusely abnormal white matter in patients with multiple sclerosis assessed with quantitative MR. PLoS One 2014;9:e95161 CrossRef Medline

18. Blystad I, Hakansson I, Tisell A, et al. Quantitative MRI for analysis of active multiple sclerosis lesions without gadolinium-based contrast agent. AJNR Am J Neuroradiol 2016;37:94-100 CrossRef Medline

19. Granberg T, Uppman M, Hashim F, et al. Clinical feasibility of synthetic MRI in multiple sclerosis: a diagnostic and volumetric validation study. AJNR Am J Neuroradiol 2016;37:1023-29 CrossRef Medline

20. McDonald WI, Compston A, Edan G, et al. Recommended diagnostic criteria for multiple sclerosis: guidelines from the International Panel on the Diagnosis of Multiple Sclerosis. Ann Neurol 2001;50: 121-27 Medline

21. Polman CH, Reingold SC, Edan G, et al. Diagnostic criteria for multiple sclerosis: 2005 revisions to the "McDonald Criteria." Ann Neurol 2005;58:840-46 Medline

22. Polman CH, Reingold SC, Banwell B, et al. Diagnostic criteria for multiple sclerosis: 2010 revisions to the McDonald criteria. Ann Neurol 2011;69:292-302 CrossRef Medline

23. Seewann A, Vrenken H, van der Valk P, et al. Diffusely abnormal white matter in chronic multiple sclerosis: imaging and histopathologic analysis. Arch Neurol 2009;66:601-09 Medline

24. Kurtzke JF. A new scale for evaluating disability in multiple sclerosis. Neurology 1955;5:580-83 Medline

25. Gabr RE, Sun X, Pednekar AS, et al. Automated patient-specific optimization of three-dimensional double-inversion recovery magnetic resonance imaging. Magn Reson Med 2016;75:585-93 CrossRef Medline

26. Redpath TW, Smith FW. Technical note: use of a double inversion recovery pulse sequence to image selectively grey or white brain matter. Br J Radiol 1994;67:1258-63 Medline

27. Traboulsee A, Simon JH, Stone L, et al. Revised recommendations of the Consortium of MS Centers Task Force for a standardized MRI protocol and clinical guidelines for the diagnosis and follow-up of multiple sclerosis. AJNR Am J Neuroradiol 2016;37:394-401 CrossRef Medline

28. Wattjes MP, Rovira À, Miller D, et al; MAGNIMS study group. Evidence-based guidelines: MAGNIMS consensus guidelines on the use of MRI in multiple sclerosis-establishing disease prognosis and monitoring patients. Nat Rev Neurol 2015;11:597-606 CrossRef Medline 